\title{
THE EQUIVARIANT COVERING HOMOTOPY PROPERTY FOR DIFFERENTIABLE $G$-FIBRE BUNDLES
}

\author{
EDWARD BIERSTONE
}

Let $G$ be a compact Lie group, and $X$ a differentiable $G$-manifold. If $p: E \rightarrow X$ is a differentiable fibre bundle, and $G$ acts differentiably on $E$ so that each $g \in G$ operates as a bundle map, then we call $p$ a differentiable $G$ fibre bundle. We show that if $p$ is a differentiable $G$-fibre bundle with Lie structure group or compact fibre, then it has the equivariant covering homotopy property. This generalizes the fact that a differentiable family of actions of a compact Lie group on a compact differentiable manifold is locally trivial.

We give some basic definitions in $\S 1$, and in $\S 2$ show that if $X$ is a $G$ manifold and $E \rightarrow X$ a differentiable fibre bundle with Lie structure group $H$ and associated principal bundle $P \rightarrow X$, then differentiable actions of $G$ on $E$ as a group of bundle maps are in natural one-one correspondence with such actions on $P$. In $\S 3$ we establish the equivariant covering homotopy property for differentiable $G$-fibre bundles with compact Lie structure group, and show that if $p: E \rightarrow X$ is a differentiable $G$-fibre bundle with connected semi-simple Lie structure group $H$, then $p$ can be reduced to a compact subgroup of $H$ so that $G$ still operates as a group of bundle maps, and hence $p$ also has the equivariant covering homotopy property. Then in $\S 4$ we define a notion of equivariant local triviality for $G$-fibre bundles, which implies the equivariant covering homotopy property, and show that any differentiable $G$-fibre bundle with Lie structure group or compact fibre is $G$-locally trivial. We conclude with some remarks relating $G$-local triviality to the equivalence of nearby differentiable actions of a compact Lie group.

The author is happy to acknowledge the generous advice of his thesis advisor, Richard S. Palais, and also some helpful conversations with Arthur G. Wasserman.

\section{Basic definitions}

Let $G$ be a topological group. A $G$-space is a Hausdorff space $X$ together with a continuous action of $G$ on $X$, i.e., a continuous map $(g, x) \rightarrow g x$ of $G \times X$ into $X$ such that $g_{1}\left(g_{2} x\right)=\left(g_{1} g_{2}\right) x$ for all $g_{1}, g_{2} \in G, x \in X$, and $1 x=x$,

Communicated by R. S. Palais, August 7, 1972. Supported by a postgraduate scholarship of the National Research Council of Canada. 
where 1 is the identity element of $G$. If $G$ is a Lie group, then a (differentiable) $G$-manifold is a differentiable $\left(C^{\infty}\right)$ manifold $X$ together with a differentiable action of $G$ on $X$. The action is effective if whenever $g x=x$ for some $g$ and all $x$, then $g=1$.

Let $G$ be a compact Lie group, and $X$ a differentiable $G$-manifold. Let $G_{x}$ be the isotropy subgroup of a point $x \in X$. The map $G / G_{x} \rightarrow X$ defined by $g G_{x} \rightarrow g x$ is an equivariant embedding whose image is the orbit $G x$. Let $V_{x}=$ $T X_{x} / T(G x)_{x}$ be the normal space to the orbit $G x$ at the point $x$. For $g \in G_{x}$, the differential of $g: X \rightarrow X$ induces an automorphism of $V_{x}$, so we have a representation $G_{x} \rightarrow G L\left(V_{x}\right)$, called a slice representation. The slice bundle $G \times{ }_{G_{x}} V_{x}$ is the $G$-vector bundle constructed from the product $G \times V_{x}$ by identifying $\left(g h, h^{-1} v\right)$ with $(g, v)$ for all $g \in G, h \in G_{x}, v \in V_{x}$; we let [g,v] denote the image of $(g, v)$ in $G \times{ }_{G_{x}} V_{x}$ under the identification map. Using the identification $G / G_{x} \rightarrow G x$, we can identify the slice bundle $G \times{ }_{G_{x}} V_{x}$ with the normal bundle of $G x$ in $X$ by the map $[g, v] \rightarrow g v$. Hence, by an equivariant version of the tubular neighborhood theorem, there is an equivariant diffeomorphism from $G \times{ }_{G_{x}} V_{x}$ onto a $G$-invariant open neighborhood of $G x$ in $X$, mapping the zero section $G / G_{x}$ canonically onto the orbit $G x$. We call the image of $V_{x}$ a slice at $x$.

A fibre bundle is a continuous map $p: E \rightarrow X$ of a Hausdorff space $E$ onto a Hausdorff space $X$ such that $p$ is locally trivial. Let $p_{i}: E_{i} \rightarrow X_{i}, i=1,2$, be two fibre bundles. A bundle map from $p_{1}$ to $p_{2}$ is a continuous map $F: E_{1} \rightarrow E_{2}$ which carries each fibre homeomorphically onto a fibre. The induced map $f: X_{1} \rightarrow X_{2}$ is clearly continuous. If $X_{1}=X_{2}$ and the induced map is the identity (so that $F$ is a homeomorphism), then the bundle map is called an equivalence.

We also consider fibre bundles with a specified structure group which acts effectively on the typical fibre. When a structure group is specified, bundle maps are understood to be induced by principal bundle maps between the associated principal bundles. Note that if $p_{i}: E_{i} \rightarrow X_{i}, i=1,2$, are fibre bundles with structure group the identity and fibre $Y$, then $E_{i}$ is equivalent to $X_{i} \times Y, i=1,2$, and over each map $f: X_{1} \rightarrow X_{2}$ of the bases there is only one bundle map, corresponding to $f \times$ id: $X_{1} \times Y \rightarrow X_{2} \times Y$.

We will mainly be concerned with differentiable $\left(C^{\infty}\right)$ fibre bundles. In this case the spaces are differentiable manifolds, the maps are $C^{\infty}$, and a structure group is a Lie group acting differentiably (from the left) on the typical fibre (and so differentiably from the right on the total space of the associated principal bundle).

\section{2. $G$-fibre bundles}

In the remainder of this paper $G$ will denote a compact Lie group.

Let $X$ be a $G$-space, and $p: E \rightarrow X$ a fibre bundle over $X$. If there is a 
continuous action of $G$ on $E$ such that each $g \in G$ operates as a bundle map over the given map $g: X \rightarrow X$ (hence, in the case that $p$ has a specified structure group, is induced by a principal bundle map), then we say that $G$ acts on $p: E \rightarrow X$ as a group of bundle maps and that $p$ is a $G$-fibre bundle (differentiable if $p$ is a differentiable fibre bundle and $X, E$ are $G$-manifolds). Note that $p$ is equivariant. A $G$-fibre bundle map (resp. $G$-fibre bundle equivalence) is a map (resp. equivalence) of $G$-fibre bundles which is equivariant with respect to the actions of $G$.

Example 1. Let $X, Y$ be $G$-spaces. $G$ acts on $X \times Y$ by $g(x, y)=(g x, g y)$ for $g \in G,(x, y) \in X \times Y$. The projection $p: X \times Y \rightarrow X$ is equivariant, and $G$ acts as a group of bundle maps if we consider $p$ as a trivial fibre bundle with structure group $G$. We call $p$ a trivial $G$-fibre bundle.

Example 2. A $G$-vector bundle is a $G$-fiber bundle with structure group a general linear group. The results in this paper are given by Segal [7] for $G$ vector bundles over compact spaces, and by Wasserman [8] for differentiable $G$-vector bundles over $G$-manifolds.

Proposition 2.1. Let $p_{i}: E_{i} \rightarrow X_{i}, i=1,2$, be G-fibre bundles with the same structure group and fibre. If $f: X_{1} \rightarrow X_{2}$ is equivariant, then the induced bundle $f^{*} E_{2}$ over $X_{1}$ is naturally a $G$-fibre bundle, and the induced map $f^{*} E_{2}$ $\rightarrow E_{2}$ is a G-fibre bundle map. If $F: E_{1} \rightarrow E_{2}$ is a G-fibre bundle map over $f$, then $E_{1}$ is $G$-equivalent to $f^{*} E_{2}$, and $F$ is the composition of a $G$-equivalence $E_{1} \rightarrow f^{*} E_{2}$ and the induced map $f^{*} E_{2} \rightarrow E_{2}$.

The proof is clear.

Now let $P \rightarrow X$ be a differentiable principal bundle with structure group a Lie group $H$. Let $Y$ be an effective $H$-manifold, and $E=P \times_{H} Y \rightarrow X$ the bundle with fibre $Y$ associated to $P$. In other words $E$ is obtained from the product $P \times Y$ by identifying $(p, y)$ with $\left(p h, h^{-1} y\right)$ for all $p \in P, y \in Y, h \in H$, and the projection $E \rightarrow X$ is induced by the projection $P \rightarrow X$. Since $H$ acts effectively on $Y$, there is a one-one correspondence between actions of $G$ as a group of bundle maps of $E \rightarrow X$ and actions as a group of bundle maps of $P \rightarrow X$; we just take, for the operation of each element of $G$ as a bundle map $E \rightarrow X$, the associated bundle map from $P \rightarrow X$ to itself, and vice-versa. If $G$ acts differentiably as a group of bundle maps of $P \rightarrow X$, then the induced action on $E \rightarrow X$ is differentiable. Conversely, we have

Theorem 2.2. If $G$ acts differentiably as a group of bundle maps of $E \rightarrow X$, then the induced action on the associated principal bundle $P \rightarrow X$ is differentiable.

Proof. Let $g_{0} \in G, x_{0} \in X$. Choose neighborhoods $U$ of $x_{0}$ in $X, V$ of $g_{0} x_{0}$ in $X$, and $W$ of $g_{0}$ in $G$ such that the bundle $E \rightarrow X$ is trivial over $U$ and $V$, and $W \cdot U \subseteq V$. With respect to trivializations $U \times Y, V \times Y$ of $E=P \times{ }_{H} Y$ over $U, V$, the action of elements of $G$ contained in $W$ is given by a $C^{\infty}$ map $W \times U \times Y \rightarrow V \times Y$, taking $(g, u, y) \in W \times U \times Y$ into $(g u, \alpha(u, g) y)$, where $\alpha(u, g) \in H$. We must show that the map $\alpha: U \times W \rightarrow H$ is $C^{\infty}$. 
Since $H$ acts effectively on $Y$, there is a finite subset $\left\{y_{1}, \cdots, y_{n}\right\}$ of $Y$ such that the Lie subgroup $\left\{h \in H \mid h y_{i}=y_{i}, i=1, \cdots, n\right\}$ of $H$ is zero-dimensional (see Gleason and Palais [2, Th. 8. 2, p. 646]). Let $H$ act on $Z=Y \times \cdots \times Y$ ( $n$ copies) by the given action on each factor, and let $z=\left(y_{1}, \cdots, y_{n}\right) \in Z$. Then the isotropy subgroup $H_{z}$ is zero-dimensional. Now the map $U \times W \rightarrow Z$ taking $(u, g) \in U \times W$ into $\alpha(u, g) z \in Z$ is $C^{\infty}$, and the image of this map lies in the orbit $\mathrm{Hz}$, which is diffeomorphic to $H / H_{z}$. In other words, the map $U \times W \rightarrow H / H_{z}$ taking $(u, g)$ to $\alpha(u, g) H_{z}$ is $C^{\infty}$., Since $H_{z}$ is zero-dimensional, then $\alpha: U \times W \rightarrow H$ is $C^{\infty}$.

\section{The equivariant covering homotopy property for differentiable $G$-fibre bundles reducible to a compact Lie structure group}

Using Theorem 2.2 we can prove the following theorem and corollary in the same way they are proved by Wasserman $[8$, Th. 2.4 , Cor. $2.5, \mathrm{p}$. 134] in the case where the structure group is an orthogonal group. I denotes the interval $[0,1]$, and $G$ always acts trivially on $I$.

Theorem 3.1. Let $G$ be a compact Lie group, and $E \rightarrow X \times I$ a differentiable G-fibre bundle with structure group a compact Lie group $H$. Then there is a differentiable $G$-fibre bundle equivalence $E \rightarrow(E \mid X \times 0) \times I$.

Corollary 3.2. If $E \rightarrow X$ is a differentiable $G$-fibre bundle with compact Lie structure group, and $f_{0}, f_{1}: Y \rightarrow X$ are $G$-homotopic (resp. differentiably $G$ homotopic) $G$-maps from a differentiable $G$-manifold $Y$ to $X$, then the induced bundles $f_{0}^{*} E$ and $f_{1}^{*} E$ are $G$-equivalent (resp. differentiably $G$-equivalent).

Using Corollary 3.2, we easily deduce the following equivariant covering homotopy property for differentiable $G$-fibre bundles with compact Lie structure group.

Corollary 3.3. Let $E_{i} \rightarrow X_{i}, i=1,2$, be differentiable $G$-fibre bundles having the same fibre and structure group, a compact Lie group. Let $F_{0}: E_{1} \rightarrow$ $E_{2}$ be a $G$-fibre bundle map over $f_{0}: X_{1} \rightarrow X_{2}$, and $f: X_{1} \times I \rightarrow X_{2}$ be a $G$ homotopy of $f_{0}$. Then there is a G-homotopy of $F_{0}$, which is a $G$-fibre bundle map $F: E_{1} \times I \rightarrow E_{2}$ over $f$. Moreover, if $F_{0}$ is differentiable and $f$ is a differentiable homotopy, then there is a differentiable covering homotopy $F$.

The above results clearly hold as well for any differentiable $G$-fibre bundle whose structure group can be reduced to a compact Lie group so that $G$ still acts as a group of bundle maps on the reduced bundle. The following theorem then shows that a differentiable $G$-fibre bundle whose structure group is a connected semi-simple Lie group has the equivariant covering homotopy property.

Theorem 3.4. Let $G$ be a compact Lie group, and $E \rightarrow X$ a differentiable G-fibre bundle with structure group a connected semi-simple Lie group $H$. Then the structure group of $E \rightarrow X$ can be reduced to a compact subgroup of $H$ so that $G$ still acts as a group of bundle maps on the reduced bundle.

Remark. In the case that $H$ is a general linear group, such a reduction to 
the orthogonal group can be given by a $G$-invariant Riemannian metric on the associated vector bundle.

Proof of Theorem 3.4. Let $\pi: P \rightarrow X$ be the principal bundle associated with $E \rightarrow X$, and $K$ a maximal compact subgroup of $H$ (assume $H$ is not compact). It suffices to find a $G$-equivariant section of the bundle $P / K=$ $P \times_{H}(H / K) \rightarrow X$.

The homogeneous space $H / K$ with any $H$-invariant Riemannian metric is a complete simply-connected Riemannian manifold of negative curvature, so that for each $h \in H$ the exponential map at $h K \in H / K$ is a diffeomorphism from the tangent space $T(H / K)_{h K}$ onto $H / K$ (Helgason [3, Chap. I, Th. 13.3]). Now $P \times_{H} T(H / K)$ is a $G$-vector bundle over $P / K$, called the tangent bundle along the fibres, and the exponential map for $T(H / K)$ induces a $G$-equivariant map $P \times_{H} T(H / K) \rightarrow P / K$, taking $\left[p h, h^{-1} v\right]$ (where $p \in P, h \in H, v \in T(H / K)$ ) into $\left[p h, \exp \left(h^{-1} v\right)\right]=\left[p h, h^{-1} \exp v\right]$.

For each $x \in X$ the isotropy subgroup $G_{x}$ acts on the fibre $H / K$ of $P / K$ over $x$ via a homomorphism $G_{x} \rightarrow H$. Since all maximal compact subgroups of $H$ are conjugate (Helgason [3, Chap. VI, Th. 2.2]), the image of this homomorphism is contained in $h K h^{-1}$ for some $h \in H$, so that $h K$ is a fixed point for the action of $G_{x}$ on $H / K$. Since $\pi$ induces a submersion of $P / K$ onto $X$, there is a $G_{x}$-equivariant section $\sigma$ of $P / K$ defined on some slice $V_{x}$ for $X$ at $x$, with $\sigma(x)=h K$. We have then a pull-back diagram:

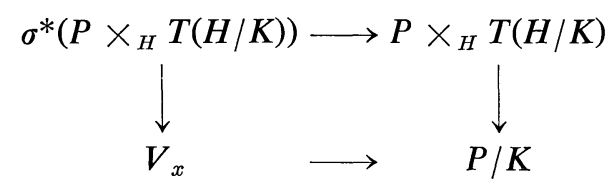

so that $\sigma^{*}\left(P \times_{H} T(H / K)\right)$ is a $G_{x}$-vector bundle over $V_{x}$, and the exponential map $\sigma^{*}\left(P \times_{H} T(H / K)\right) \rightarrow(P / K) \mid V_{x}$ is a $G_{x}$-equivariant fibre-preserving diffeomorphism.

We now construct a $C^{\infty}$ equivariant section of $P / K \rightarrow X$. For each $x \in X$, shrink $V_{x}$ equivariantly to $U_{x}, \mathrm{Cl}\left(U_{x}\right) \subset V_{x}(\mathrm{Cl}=$ closure), and choose a countable number of points $x(1), x(2), \cdots$ such that the slice neighborhoods $G \cdot U_{x(i)}$ of the orbits $G x(i)$ cover $X$. Set $A_{0}=\emptyset$, and define $A_{n}$ inductively by $A_{n}=G \cdot \mathrm{Cl}\left(U_{x(n)}\right) \cup A_{n-1}$. Suppose $C^{\infty}$ equivariant sections $s_{i}$ of $P / K \rightarrow X$ are defined on $A_{i}$ for $i<n$, such that $s_{i} \mid A_{i-1}=s_{i-1}$. Since there is a $G_{x(n)}$ equivariant fibre-preserving diffeomorphism from $(P / K) \mid V_{x(n)}$ to a $G_{x(n)}$ vector bundle over $V_{x(n)}, s_{n-1}$ extends to a $C^{\infty}$ equivariant section $s_{n}$ over $A_{n}$. Define $s$ by $s(x)=s_{n}(x)$ for $x \in A_{n}-A_{n-1}$. Since $X$ is the union of the interiors of the $A_{n}$, we see $s$ is a $C^{\infty}$ equivariant section $X \rightarrow P / K$.

\section{G-local triviality and the equivariant covering homotopy property}

Let $p: E \rightarrow X$ be a differentiable $G$-fibre bundle. We say $p$ is $G$-locally trivial 
if for each $x \in X$ there is a $G_{x}$-invariant neighborhood $U_{x}$ of $x$ such that $p \mid U_{x}$ is differentiably $G_{x}$-equivalent to the trivial $G_{x}$-fibre bundle $U_{x} \times p^{-1}(x)$.

By the equivariant covering homotopy property (Corollary 3.3), a differentiable $G$-fibre bundle with structure group a compact or semi-simple Lie group is $G$-locally trivial. On the other hand, the following theorem implies that if $p: E \rightarrow X$ is a differentiable $G$-fibre bundle which is $G$-locally trivial, then $p$ has the equivariant covering homotopy property.

Theorem 4.1. Let $G$ be a compact Lie group, and $p: E \rightarrow X \times I$ a differentiable $G$-fibre bundle which is $G$-locally trivial ( $G$ acts trivially on $I$ ). Then there is a differentiable G-fibre bundle equivalence $E \rightarrow(E \mid X \times 0) \times I$ (the map is understood to be induced by a principal bundle map in the case that $p$ is a G-fibre bundle with Lie structure group $H$ ).

Proof. The proof is similar to that of the equivariant covering homotopy property for locally trivial fibre spaces given, for example, in Husemoller [4, pp. 49-51]. We choose a locally finite countable invariant covering $G \cdot U_{i}$ of $X$ such that $U_{i}$ is a slice at $x(i), i=1,2, \cdots$, and there is a $G_{x(i)}$-equivalence $h_{i}: U_{i} \times I \times Y_{i} \rightarrow E \mid\left(U_{i} \times I\right)$, where $Y_{i}=p^{-1}(x(i))$ (when $p$ has structure group $H, G_{x(i)}$ acts on the $H$-manifold $Y_{i}$ by a homomorphism $G_{x(i)} \rightarrow H$ ).

There is a $G$-invariant $C^{\infty}$ map $u_{i}: X \rightarrow[0,1]$ such that $u_{i}^{-1}(0,1] \subseteq G \cdot U_{i}$ and $\max _{i} u_{i}(x)=1$ for all $x \in X$. Define $G$-fibre bundle equivalences

$$
k_{i}: G \times_{G_{x(i)}}\left(U_{i} \times I \times Y_{i}\right) \rightarrow E \mid\left(G \cdot U_{i} \times I\right)
$$

by $k_{i}[g,(u, t, y)]=g h_{i}(u, t, y)$ for $g \in G, u \in U_{i}, t \in I, y \in Y_{i}$, and define $G$ fibre bundle maps

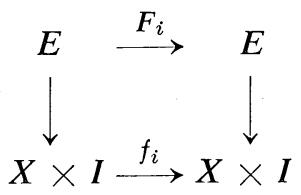

as follows :

$$
\begin{gathered}
f_{i}(x, t)=\left(x, t\left(1-u_{i}(x)\right)\right), \quad(x, t) \in X \times I ; \\
F_{i}=\text { id outside } p^{-1}\left(G \cdot U_{i} \times I\right) ; \\
F_{i} \circ k_{i}[g,(u, t, y)]=k_{i}\left[g,\left(u, t\left(1-u_{i}(u)\right), y\right)\right], \\
{[g,(u, t, y)] \in G \times_{G_{x(i)}}\left(U_{i} \times I \times Y_{i}\right) .}
\end{gathered}
$$

Then $F=\cdots \circ F_{3} \circ F_{2} \circ F_{1}$ is a $G$-fibre bundle map over $f=\ldots \circ f_{3} \circ f_{2} \circ f_{1}(F$, $f$ are well-defined since all but a finite number of terms in the infinite compositions are equal to the identity near any point).

By Proposition $2.1, E$ is $G$-equivalent to $f^{*} E$, which is $G$-equivalent to $(E \mid X \times 0) \times I$ by the definiton of $f$. This completes the proof. 
Using Theorem 4.1, we can now deduce the equivariant covering homotopy property for a differentiable $G$-fibre bundle with structure group any Lie group:

Theorem 4.2. Let $G$ be a compact Lie group, and $E \rightarrow X$ a differentiable $G$-fibre bundle with structure group a Lie group $H$. Then $E$ is $G$-locally trivial.

Proof. Let $\pi: P \rightarrow X$ be the associated principal bundle, and let $x \in X$. The isotropy subgroup $G_{x}$ acts on the fibre $H$ of $P$ over $x$ via a homomorphism $\alpha: G_{x} \rightarrow H$. Consider the bundle $P / \alpha\left(G_{x}\right)$ with fibre $H / \alpha\left(G_{x}\right)$ associated with $P$. The point $1 \alpha\left(G_{x}\right)$ in the fibre over $x$ is a fixed point for the action of $G_{x}$. Since $P / \alpha\left(G_{x}\right) \rightarrow X$ is an equivariant submersion onto $X$, there is a $G_{x}$-equivariant section $\sigma$ of $P / \alpha\left(G_{x}\right)$ defined on some $G_{x}$-invariant neighborhood $U_{x}$ of $x$, which is $G_{x}$-contractible to $x$.

Hence $E \mid U_{x}$ can be reduced to the compact subgroup $\alpha\left(G_{x}\right)$ of $H$ so that $G_{x}$ still acts as a group of bundle maps. The result now follows from the equivariant covering homotopy property for $G$-fibre bundles with compact Lie structure group (Corollary 3.3).

We conclude with some remarks relating $G$-local triviality to the equivalence of nearby differentiable actions of a compact Lie group.

If $p: E \rightarrow X$ is any differentiable $G$-fibre bundle with compact fibre, then we can obtain the equivariant covering homotopy property for $p$ by proving an analogue of Theorem 3.1. Hence $p$ is also $G$-locally trivial.

Definitions. Let $G$ be a compact Lie group, and $X, Y$ two differentiable manifolds. A differentiable family of actions of $G$ on $Y$ parametrized by $X$ is a differentiable map $\Phi: X \times G \times Y \rightarrow Y$ such that for each $x \in X$ the map $\Phi_{x}: G \times Y \rightarrow Y$ taking $(g, y) \in G \times Y$ into $\Phi(x, g, y)$ is a differentiable action of $G$ on $Y$. This family is said to be locally trivial at $x_{0} \in X$ if there are an open neighborhood $U$ of $x_{0}$ in $X$ and a differentiable map $\Psi: U \times Y \rightarrow Y$ such that:

1. for each $x \in U$ the map $\Psi_{x}: Y \rightarrow Y$ taking $y$ into $\Psi(x, y)$ is a diffeomorphism of $Y$, and $\Psi_{x_{0}}=\mathrm{id}_{Y}$;

2. $\Phi(x, g, \Psi(x, y))=\Psi\left(x, \Phi\left(x_{0}, g, y\right)\right)$ for each $x \in U, g \in G$, and $y \in Y$.

A family of differentiable actions of $G$ on $Y$ is said to be locally trivial if it is locally trivial at each point $x$ of the parameter space $X$.

Now if $p: E \rightarrow X$ is a product bundle $X \times Y \rightarrow X$ with compact fibre $Y$, and $G$ acts on $E$ as a group of bundle maps with the induced action on $X$ trivial, then the $G$-local triviality of $p$ is just a restatement of the fact that a differentiable family of actions of a compact Lie group on a compact manifold $Y$ is locally trivial (Palais [5], Calabi [1]).

The conjecture of Calabi [1, p. 213] that such a family is locally trivial even when $Y$ is not compact had already been shown to be false by Palais and Stewart [5], [6]. This shows that a differentiable $G$-fibre bundle $p: E \rightarrow X$ with noncompact fibre does not in general have the equivariant covering homotopy propery. (We note here also the observation of Palais and Stewart that a con- 
tinuous family of actions of a compact Lie group on a compact space $Y$ is not in general locally trivial. Hence, though Theorem 4.1, for example, is valid in the continuous case (when $X$ is paracompact), we cannot hope for an equivariant covering homotopy property for a broad class of continuous $G$-fibre bundles.)

From the $G$-local triviality of $G$-fibre bundles with Lie structure group (Theorem 4.2), we deduce, however, the following result.

Theorem 4.3. Let $H$ be a Lie group, and $Y$ an H-manifold. Let $\Phi: X \times$ $G \times Y \rightarrow Y$ be a differentiable family of actions of a compact Lie group $G$ on $Y$ such that for each $x \in X$, there is a homomorphism $\varphi_{x}: G \rightarrow H$, and $\Phi(x, g, y)=\varphi_{x}(g) y$ for all $g \in G, y \in Y$. Then $\Phi$ is locally trivial.

As a final remark we note a type of action of a compact Lie group $G$ on a differentiable fibre bundle with Lie structure group which is more general than an action as a group of bundle maps and for which the equivariant covering homotopy property still holds. Let $\pi: P \rightarrow X$ be a differentiable principal bundle with Lie structure group $H$, on which $G$ acts as a group of bundle maps, and let $Y$ be an effective $H$-manifold. If $G$ acts (on the left) on $Y$ commuting with the the action of $H$, there is an induced action on the total space $E=P$ $\times_{H} Y$ of the associated bundle with fibre $Y$ given by $g[p, y]=[g p, g y]$, with respect to which the projection $E \rightarrow X$ is equivariant. The equivariant covering homotopy property for $\pi$ clearly implies that for $E \rightarrow X$. This also gives a generalization of Theorem 4.3: with the same notation as in Theorem 4.3, if $G$ acts on $Y$ commuting with the action of $H$, and $\Phi$ is given by $\Phi(x, g, y)=$ $\varphi_{x}(g) g y$, then $\Phi$ is locally trivial.

\section{References}

[1] E. Calabi, On differentiable actions of compact Lie groups on compact manifolds, Proc. Conf. on Transformation Groups (New Orleans, 1967), Springer, New York, 1968, 210-213.

[2] A. M. Gleason \& R. S. Palais, On a class of transformation groups, Amer. J. Math. 79 (1957) 631-648.

[ 3 ] S. Helgason, Differential geometry and symmetric spaces, Academic Press, New York, 1962.

[4] D. Husemoller, Fibre bundles, McGraw-Hill, New York, 1968.

[5] R. S. Palais, Equivalence of nearby differentiable actions of a compact group, Bull. Amer. Math. Soc. 67 (1961) 362-364.

[6] R. S. Palais \& T. E. Stewart, Deformations of compact differentiable transformation groups, Amer. J. Math. 82 (1960) 935-937.

[7] G. Segal, Equivariant K-theory, Inst. Hautes Etudes Sci. Publ. Math. No. 34 (1968) 129-151.

[ 8 ] A. G. Wasserman, Equivariant differential topology, Topology 8 (1969) 127-150. 